\title{
Corpus Callosum
}

National Cancer Institute

\section{Source}

National Cancer Institute. Corpus Callosum. NCI Thesaurus. Code C12446.

A white matter structure within the cleft that separates the left and right cerebral

hemispheres in the mammalian brain. It is composed of a wide, flat bundle of 200-250

million axonal projections. 